mus als Indicator und deren Schmelzpunkt bestimmt. Es wai

\begin{tabular}{|c|c|c|}
\hline für die Krystalle aus & $\begin{array}{l}\text { der } \\
\text { Schmelz- } \\
\text { punkt }\end{array}$ & $\begin{array}{l}\text { das Äquiva } \\
\text { lentgewicht }\end{array}$ \\
\hline $\begin{array}{l}\text { Palmölwaschwasser } \\
\text { Olivenkernölwaschwasser } \\
\text { Cottonölwaschwassor } \\
\text { Es ist für Korksäure } \\
\text { - - - Azelaïnsăure. }\end{array}$ & $\begin{array}{l}108 \\
107 \\
105,5 \\
140 \\
106\end{array}$ & $\begin{array}{l}92,4 \\
94,1 \\
94,5 \\
87,0 \\
94,0\end{array}$ \\
\hline
\end{tabular}

Die Krystalle aus allen Waschwässern gaben in Wasser gelöst, mit Natronlauge genau neutralisirt, mit

Eisenchlorid einen bräunlich-fleisebfarbenen flockigen

Silbernitrat - weissen, flockigen

Bleizucker - weissen, pulverigen Niederschlag

Kupfersulfat - blaugrünen in verd. SchwefelBariumehlorid keinen sĩure löslichen Niederschlag

Die Krystalle in überschüssigem Ammoniak gelöst gaben mit Chlorcalcium erst beim Kochen einen weissen, körnig-krystallinischen Niederschlag. Die ammoniakalische Lösung hinterlässt beim Eindampfen einen sauer reagirenden Rückstand. Über $300^{\circ}$ erhitzt, destilliren die Säuren anscheinend unzersetzt. Sie zeigen somit alle Reactionen der Azelaïnsäure.

Die Mutterlaugen von diesen Krystallen gaben beim Eindampfen breiige, gelbgefärbte, stark sauer reagirende Massen, welche leichter lösliche, vermuthlich kohlenstoffärmere Säuren enthielten. Eine Isolirung und nähere Untersuchung derselben war bei der geringen Menge der vorliegenden Proben nicht ausführbar; hingegen wurde in ibnen das beim Ranzigwerden möglicher Weise freigewordene Glycerin gesucht. $\mathrm{Zu}$ diesem Zwecke wurden sie in wenig Wasser gelöst, mit einem Überschuss gelöschten Kalkes vermischt, zur Trockene verdampft. der Rückstand mit Ätheralkohol ausgeschüttelt und die Auszüge verdunstet. Es blieb in keinem Falle ein Rückstand; Glycerin war also nicht vorhanden. Zur Sicherheit wurden auch noch die Papierstreifen, von denen das ranzige Fett mit Äther abgezogen worden, auf Glycerin geprüft. Die durch Auslaugen der Streifen mit Wasser erhaltenen Lösungen hinterliessen beim Eindampfen einen amorphen dunkelbraunen Rückstand von Huminsubstanzen. Diese haben sich vermuthlich aus der Papierfaser durch die Jahre lange Einwirkung der Säuren gebildet. Ihre wässerigeñ Lösungen mit überschüssigem Kalk zur Trockene verdampft, gaben an Ätheralkohol nichts Lösliches ab, enthielten somit ebenfalls kein Glycerin.

Obwohl nun vorstehende Untersuchung keineswegs vollständig geuannt werden kann, da dieselbe mit zu geringen Substanzmengen begonnen und auf die flüchtigen Fettsäuren gar keine Rücksicht genommen worden ist, so geht doch daraus hervor, dass man sich das lianzigwerden der Fette so vorzustellen hat, dass dieselben wahrscheinlich durch Wasser in Fettsäuren und Glycerin zerlegt, diese aber gleichzeitig durch den Luftsauerstoff oxydirt werden. Die Oxydation muss sich sowohl auf die Fettsäuren, als auch auf das Glycerin erstrecken, da letzteres im freien Zustande nicht nachgewiesen werden kann. Die Fettsäuren zerfallen dabei in kohlenstoffärmere, sauerstoffreichere Säuren, welche zum Theil der Fettsäurereihe, zum Theil aber der Oxalsäurereihe angehören, aus welcher namentlich Azelaïnsäure, die auch bei der künstlichen Oxydation der Fettsäuren mit Salpetersäure stets auftritt, hervorzuheben ist.

Brünn, Januar 1889.

Laboratorium der deutschen Staatsgewerbeschule.

\section{Die Öle des Delphinus phoecaena}

(Meerschweines).

$$
\text { Von }
$$

Chr. Steenbuch.

Bei einer Untersuchung der verschiedenen Fettstoffe, mit welcher ich im Vereine mit Hrn. Assistent Dethlefsen behufs einer Kritik der üblichen Butteruntersuchungsmethoden beschäftigt bin, habe ich Gelegenheit gehabt, verschiedene Öle des Meerschweines einer vorläufigen Untersuchung zu unterwerfen.' Das Untersuchungsmaterial habe ich von der "Middelfart Trankogeri ${ }^{“}$ bekommen. Schon seit dem Mittelalter wird hier (im "Kleine Belt") der Fang dieser Thiere betrieben.

Durch die von Chevreul i. J. 1817 (Ann. chim. 7. S. 264) veröffentlichte Untersuchung ist das Vorhandensein beträchtlicher Mengen von Valeriansäure im Thrane des Delphinus globiceps (Grind-Wallfisch) bekannt, und derselbe Verfasser hat auch diese Säure in anderen Thransorten, obwohl in geringeren Mengen, nachgewiesen. Salkowski (Z. anal. 26 S. 564) hat nach Unsuchung mehrerer Medicinalthransorten die Auffassung ausgesprochen, dass die gewöhnliche Angabe, der Leberthran enthalte Glyceride niederer Fettsäuren, für die heutzutage im Handel vorkommenden Medicinalthrane keine Geltung mehr hat, indem er vermuthet, dass es sich auch früher nicht 
um das Vorkommen von Glyceriden dieser Säuren gehandelt hat, sondern um die Säuren selbst, deren Quelle er in den Fäulnissproducten der Eiweissstoffe der Leber sucht. Für die gereinigten Medicinal-Leberthransorten stimmen meine bisherigen Untersuchungen mit denen Salkowski's überein.

Für alle Thransorten aber hat diese Annahme nicht Geltung, indem die vorläufige Untersuchung der Meerschweinöle einen sehr beträchtlichen Gehalt der Ätherarten flüchtiger Säuren gezeigt hat. Die zur Untersuchung vorliegenden Sorten waren der gewöhnliche Meerschweinthran von brauner Farbe und ein goldgelbes $\ddot{O}]$, welches aus dem Kiefer des Meerschweines gewounen wird.

Nach der Wollny'schen Abart des Reichert'schen Verfahrens untersucht, rerlaugte das Destillat von $5 \mathrm{~g}$ des gemeinen Meerschweinthranes 46,9 ec Zehntel-Barytlösung zur Neutralisation der im Destillate gelösten flüchtigen Süuren, während, in derselben Weise behandelt, $5 \mathrm{~g}$ des Öles aus dem Kiefer 131,6 ce verbrauchten. Es sind also sehr beträchtliche Mengen von flüchtigen Säuren in diesen Sorten enthalten ${ }^{1}$ ). Valeriansäure ist im Destillate durch den Geruch erkennbar; es scheint aber, als ob eine Reihe von Säuren mit niedriger Kohlenstoffmenge enthalten sind. Die Isovaleriansäure ist bei $20^{\circ}$ in $23 \mathrm{Th}$. Wasser löslich. Nach dem Alkaliverbrauche berechnet, sollte in den $110 \mathrm{cc}$ des Destillates $1,34 \mathrm{~g} \mathrm{Va}$ leriansäure enthalten sein, und falls es sich nur um Valeriansäure handelte, würde im Destillate eine mehr als hinlängliche Wassermenge vorhanden sein, um diese Säure aufzulösen. Es war dies indessen nicht der Fall. Ebenso wie beim Destillate von Cocosnussöl schwimmen auf dem Destillate beträchtliche Mengen öliger Tropfen flüchtiger, flüssiger im Wasser schwerlöslicher Säuren, welche vor der Titrirung abfiltrirt wurden. $\mathrm{Zu}$ einem Versuche wurde das mit diesen Säuren beladene Filter wieder mit $140 \mathrm{cc}$ Wasser destillirt, und die $110 \mathrm{cc}$ neuen Destillats erforderten dann, nach Abfiltriren der noch ungelöst gebliebenen flüssigen Säuren, 12,1 cc Zehntel-Barytlösung zur Neutralisation.

Berechnet man nur die wach Reichert gewonnenen, im Wasser löslichen Säuren als Valeriansäure, so würde dieses im Öle aus dem Kiefer einem Gehalte von 26,8 Proc.,

1) Herr Professor Stein, Kopenhagen, hat mir freundlichst mitgetheilt, dass in "The aunual Report of the New York State Dairy Commissioners. 1886" ähnliche Sorten "Marine Oils" mit ebenso hobem Gehalte fluchtiger Săuren erwähnt sind. im gemeinen Meerschweinsthran von etwa 10 Proc. entsprechen.

Sind dagegen Säuren mit höherem Kohlen: stoffgehalt vorhanden, so würde der procentische Gehalt dieser Säuren noch grösser sein; hierzu kommt ferner, dass man, wie bekannt, bei der Reichert'schen Methode bei Weitem nicht alle fiüchtigen Säuren im Destillate bekommt.

Es scheint mir, als ob die erwähnte Beobachtung Bedeutung für die jetzt allgemein gebräuchlichen Butteruntersuchungsverfahren hat. Schon die Schwankungen in der Zusammensetzung des natürlichen Butterfettes können zu Schwierigkeiten beim Erkennen einer Fälschung mit geringen Mengen von Fettmischungen Veranlassung geben, aber noch schwieriger wird es, wenn es sich zeigen sollte, dass natürliche Fettstoffe, welche zur Butterfälschung dienen könnten, auch beträchtliche Mengen flüchtiger, wasserlöslicher Säuren enthalten. Es würden dann, wenn solche zu Fälschungen benutzt werden, alle jetzt üblichen Butteruntersuchungsverfahren unsicher sein. Es gilt dieses von der Hehner'schen Methode, sowie von dem Verfahren von Reichert, Köttsdorfer u. A., indem diese in solchem Falle auf unrichtigen Voraussetzungen beruhen. Es kommt indessen eine grosse Menge pflanzlicher und thierischex Fettstoffe vor, deren Verhalten den bei Butteruntersuchungen angewandten Verfahren gegenüber noch nicht untersucht sind, und es scheint mir, als ob in den beim Oele des Meerschweines erhaltenen Resultaten eine Aufforderung liegt, eine möglichst vollständige Sammlung von Resultaton in dieser Richtung herbeizuschaffen.

Es sei noch bemerkt, dass ich mit Hilfe des Oeles aus dem Kiefer des Meerschweines mehrere Mischungen dargestellt habe, welche eine dem Butterfette entsprechende Menge flüchtiger Säuren enthält. Sind in diesen Mischungen nur 30 bis 40 Proc. Butterfett enthalten, so wird man Schwierigkeit haben, durch den Geruch die Valeriansäure im Gemische der anderen stark riechenden Säuren des Destillats zu erkennen.

Ein Umstand, der vielleicht zum Erkennen einer Mischung mit Oelen der Delfinsorten benutzt werden könnte, ist das Verhältniss zu Weingeist. Schon für das $\mathrm{Oel}$ des Delphinus globiceps hat Chevreul beobachtet, dass dieses bei $70^{n}$ im Weingeist leicht löslich ist und eine gleiche Erfahrung habe ich mit dem Oele aus dem Kiefer des Meerschweines gemacht. Practische Versuche in dieser Richtung habe ich indessen nọch nicht angestellt. 
Von der Anwendung des Refractometers bei der Butteruntersuchung könnte man vielleicht schon im Voraus aus theoretiocheu Grinden vermuthen, dass dieser Apparat uur in seltenen Fällen Hilfe leisten kann. Lis hat sich gezeigt, dass die oben genannten Kunstmischungen mit einem dem Butterfette entsprechenden Inhalte flüchtiger Säuren auch einen Refractionsexponenten zeigten, der innerhalb der Grenzen des beim Butterfette beobachteten liegt.

Universitatslaboratorium zu Kopenhagen.

10. December 1888.

\section{Zur Spiritusdenaturirung.}

\section{Von}

Jul. Schenkel.

Die von C. Reinhard in d. Z. 1888 S. 634 exhobenen Klagen gegen die mit dem allgemeinen Denaturirungsmittel denaturirten Spixitus sind jetzt auch in die politischen Zeitungen übergegangen und bringen den an und für sich sohon sehr unbeliebten denaturirten Spiritus noch mehr in Verruf, als er verdient.

Die Zerstörung, bez. das Angegriffenwerden der Metalltheile der Spirituslampen wird von Reinhard irrthümlicher Weise dem allgemeinen Denaturirungsmittel (einem Gemisch von 1 Raumth. Pyridinbasen mit 4 Raumth. Holzgeist) zugeschrieben. Das Pyridin löst aber weder in reinern Zustande, noch in der Verdünnung, in welcher es im denaturirten Spiritus enthalten ist (4 cc Pyridin in 1180 proc. Spiritus) Messing bez. Zink und Kupfer; ebensowenig der Holzgeist.

Die von Reinhard beobachteten achlimmen Wirkungen des denaturirten Spiritus beruhen einfach darauf, dass bis zum Juli 1888 der Geruch des denaturirten Spiritus seitems vieler Händler durch Zusatz ron Säuren und ächerischen Ölen "verbessert" wurde. Durch dieses Verfahren wurde meist nicht die beabsichtigte Neutralisirung der Pyridinbasen herbeigeführt, sondern dem Spiritus ein Ueberschuss von Säure (neist Essig- oder Schwefelsäure) zugesetzt. Dass dadurch dio Metall theile der Spirituslampen angegriffen und zerstört werden, liegt auf 'der Hand.

Durch Beschluss des Bundesrathes vom 21. Juni 1888 worde jeder Zusatz zu dem denaturirten Spiritus verboten, durch welchen das Denaturirungsmittel ganz oder theilweise aus dem Spiritus ausgeschieden, oder durch welchen die Wirkung des Denaturi- rungsmittels in Bezug auf Geschmack oder Gerucb verändert wird; als Ausnahme ist ein Zusatz von gewissen ätherischen Ölen zum allgemeinen Denaturirungsmittel gestattet.

Der angesäuerte Spiritus ist jetrt aus dem Haudel verschwunden, es sind demnach obige Klagen Lber deu denaturirten Spiritus hinfällig geworden.

\section{Zur Analygo von Natrium-Snlfat.}

\section{Von}

\section{Dr. Isbert \& Venator.}

Es macht sich in der Technik sehr sehr bäufig das Bedürfniss nach einer sicheren, und dabei ror allen Dingen möglichst schnellen Bestimmung des Gehaltes an schwefelsaurem Natron in käuflichem Sulfat geltend Zur Werthschätzung eines solchen ist die Kenntniss des Gehaltes an Natriumsulfat unbedingt erforderlich und vor Allem baben die Glasfabrikanten ein grosses Interesse daran, den Gehalt der ihnen zum Verkauf angebotenen Waare zu kennen. Gerade die Letzteren beweisen jedocb im Allgemeinen ihren Lieferanten gegenüber eine grosse Vertrauensseligkeit, indem sie für gewöhnlich den ibnen von diesen angegebenen Gehalt für ibre Waare ohne weitere Controle aunehmen. Fis wird diese Erscheinung wohl in den weitaus meisten Fällen auf die Kostspieligkeit, mit der eine solche Controle, d. h. eine Untersuchung des Sulfuts auf seinen Gehalt ron einer chemischen Versuchsstation, verbundeu ist, zurūckzuführen sein, da die bisher gebräuchlichste Bestimmung des Geholtes an Natriumsulfat auf einer Gesammt-Analyse des Productes beruhte. Es wurden nämlich die in dem Sulfat befindlichen fremden Beimengungen: Kochsalz, freie Schwefelsäure bez. saures schwefelsaures Natron, Eisenoxyd und Thonerde, Kalk, Magnesia und Unlosliches bestimmt, als schwefelsaure Salze berechnet und die Differenz zwischen der Summe dieser Salze und 100 als Procente schwefelsaures Natron angenommen. Dass für diese, nur mit nicht unbeträchtlichem Aufwand an Zeit und Arbeit vorzunehmende Untersuchung auch ein entsprecbendes Honorar seitens des analytischen Chemikers verlangt wird und verlangt werden muss, liegt auf der Hand.

Eine andere, ebenfalls sehr häufig ausgeführte, directe Bestimmung des Gehaltes au Sulfat besteht in gewichtsanalytischer Bestimmung der Gesaumtschwefelsäure als 